\title{
Gravidez na adolescência: Contribuições dos profissionais de saúde frente à educação sexual e reprodutiva
}

\author{
Adolescent pregnancy: Contributions of health professionals to sexual and reproductive education \\ Embarazo adolescente: Contribuciones de los profesionales de la salud a la educación sexual y
} reproductiva

Rayssa Stéfani Sousa Alves ORCID: https://orcid.org/0000-0002-9666-675X Pontifícia Universidade Católica de Goiás, Brasil

E-mail: rayssastefani02@gmail.com

Francisco Lucas Leandro de Sousa ORCID: https://orcid.org/0000-0003-2802-2378 Centro Universitário Maurício de Nassau, Brasil E-mail: lucasleandro2912@gmail.com

Airton César Leite

ORCID: https://orcid.org/0000-0001-7184-8488

Centro Universitário Santo Agostinho, Brasil E-mail: ainton.cesar2014@gmail.com

Mariana Pereira Barbosa Silva

ORCID: https://orcid.org/0000-0003-0852-8099 Universidade Estadual do Piauí, Brasil E-mail: marianapbsilvaa@gmail.com João Kelson Araújo da Silva

ORCID: https://orcid.org/0000-0002-1173-1752 Universidade Federal do Piauí, Brasil

E-mail: joaokelaonjk@gmail.com

Elielson Rodrigues da Silva

ORCID: https://orcid.org/0000-0002-9628-1809

Centro Universitário do Rio São Francisco, Brasil

E-mail: elielsonfasvipa@gmail.com

Sidney Freires dos Santos

ORCID: https://orcid.org/0000-0002-4112-3336

Centro Universitário Maurício de Nassau, Brasil

E-mail: sidneyfreire12@gmail.com

Hugo Alexandre Rodrigues

ORCID: https://orcid.org/0000-0002-1822-9896 Universidade Estadual do Ceará, Brasil E-mail: hugoalexandre13@gmail.com

Carlos Colares Maia

ORCID: https://orcid.org/0000-0003-3194-383X

Instituto Centro de Ensino Tecnológico, Brasil E-mail: carloscolaresm@yahoo.com.br

Filipe Eugênio Rodrigues Silvestre ORCID: https://orcid.org/0000-0002-3002-1051 Universidade Estadual do Ceará, Brasil E-mail: filipe-eugenio@hotmail.com

Luiz Fernando de Almeida ORCID: https://orcid.org/0000-0003-2785-587X Universidade Federal de São João del-Rei, Brasil

E-mail: luizfernando2204@yahoo.com.br

Cristiany Favalessa Sorio

ORCID: https://orcid.org/0000-0003-1582-6721

Universidade Vila Velha, Brasil

E-mail: cristianyfsorio@outlook.com

Ana Rosemere Favalessa

ORCID: https://orcid.org/0000-0001-5134-1129 Doctum, Brasil

E-mail: rose.favalessa@hotmail.com

Bruna Kely Oliveira Santos

ORCID: https://orcid.org/0000-0002-6938-5055 


\author{
Maria Augusta Oliveira Amando \\ ORCID: https://orcid.org/0000-0002-4922-8431 \\ Centro Universitário Tiradentes, Brasil \\ E-mail: mariaaugusta_23@hotmail.com \\ Leticia César Jatobá Mendonça \\ ORCID: https://orcid.org/0000-0001-6593-1568 \\ Centro Universitário Tiradentes, Brasil \\ E-mail: leticiacjm@gmail.com \\ Carolina Queiroga de Miranda \\ ORCID: https://orcid.org/0000-0001-8216-9499 \\ Centro Universitário Tiradentes, Brasil \\ E-mail: carolinaqueiroga2000@ hotmail.com \\ Jéssica Moreira Fernandes \\ ORCID: https://orcid.org/0000-0001-8888-1962 \\ Centro Universitário Católico Salesiano Auxilium, Brasil \\ E-mail: jessica-fernandes-@outlook.com \\ Lynna Stefany Furtado Morais \\ ORCID: https://orcid.org/0000-0002-5611-2736 \\ Universidade Federal do Triângulo Mineiro, Brasil \\ E-mail: lynnastefany.morais@gmail.com
}

\title{
Resumo
}

Este estudo tem como objetivo compreender as contribuições dos profissionais de saúde frente a educação sexual e reprodutiva das adolescentes. Trata-se de uma revisão integrativa de literatura, com abordagem qualitativa, realizada por meio da buscativa de artigos indexados na Biblioteca Virtual em Saúde (BVS), com o auxílio das seguintes bases de dados: Scientific Electronic Library Online (SCIELO), National Center for Biotechnology Information (PUBMED), Literatura Latino-Americana e do Caribe em Ciências da Saúde (LILACS). Os critérios de inclusão compreendem pesquisas de revisão da literatura disponíveis nos bancos de dados descritos, compreendidos entre os anos de 2000 a 2020. Como critérios de exclusão, não foram considerados artigos mediante a recompensação monetária, incompletos e não convergentes com este estudo. Após a aplicação dos critérios de inclusão e exclusão, restaram 14 artigos cientificos. Os resultados evidenciam a informação e o conhecimento inadequado dos adolescentes em relação às questões de sexualidade e prevenção da gravidez precoce, considerando a necessidade de uma educação sexual mais ampla com espaços de diálogos e discussões de dúvidas das adolescentes, tanto na escola, como no âmbito familiar. Portanto, é de grande importância que ocorra a participação e cooperação dos profissionais de saúde no processo de educação das adolescentes, promovendo o acesso à informações referente à métodos contraceptivos, conhecimento sobre as DST e orientações quanto as consequencias de uma gravidez precoce para a sua vida familiar, na escola ou na vida profissional, repercutindo sobre os projetos pessoais.

Palavras-chave: Sexualidade; Adolescência; Gravidez na adolescência; Doenças sexualmente transmissíveis.

\begin{abstract}
This study aims to understand the contributions of health professionals to the sexual and reproductive education of adolescents. It is an integrative literature review, with a qualitative approach, carried out by searching for articles indexed in the Virtual Health Library (VHL), with the help of the following databases: Scientific Electronic Library Online (SCIELO), National Center for Biotechnology Information (PUBMED), Latin American and Caribbean Literature in Health Sciences (LILACS). The inclusion criteria include literature review surveys available in the databases described, between the years 2000 to 2020. As exclusion criteria, articles were not considered for monetary reward, incomplete and not converging with this study. After applying the inclusion and exclusion criteria, 14 scientific articles remained. The results show the adolescents' inadequate information and knowledge regarding issues of sexuality and prevention of early pregnancy, considering the need for a broader sexual education with spaces for dialogues and discussions of doubts by the adolescents, both at school and in the context familiar. Therefore, it is of great importance that health professionals participate and cooperate in the education process of adolescents, promoting access to information regarding contraceptive methods, knowledge about STDs and guidelines regarding the consequences of an early pregnancy for their lives. family, school or professional life, reflecting on personal projects.
\end{abstract}

Keywords: Sexuality; Adolescence; Teenage pregnancy; Sexually transmitted diseases.

\section{Resumen}

Este estudio tiene como objetivo comprender los aportes de los profesionales de la salud a la educación sexual y reproductiva de los adolescentes. Se trata de una revisión integradora de la literatura, con enfoque cualitativo, realizada mediante la búsqueda de artículos indexados en la Biblioteca Virtual en Salud (BVS), con la ayuda de las siguientes bases de datos: Scientific Electronic Library Online (SCIELO), Centro Nacional de Información Biotecnológica (PUBMED), Literatura Latinoamericana y del Caribe en Ciencias de la Salud (LILACS). Los criterios de inclusión incluyen encuestas de revisión de la literatura disponibles en las bases de datos descritas, entre los años 
2000 a 2020. Como criterios de exclusión, los artículos no fueron considerados para recompensa monetaria, incompletos y no convergentes con este estudio. Tras aplicar los criterios de inclusión y exclusión, quedaron 14 artículos científicos. Los resultados evidencian la inadecuada información y conocimientos de las adolescentes sobre temas de sexualidad y prevención del embarazo precoz, considerando la necesidad de una educación sexual más amplia con espacios de diálogo y discusión de dudas por parte de las adolescentes, tanto en la escuela como en el contexto familiar. Por ello, es de gran importancia que los profesionales de la salud participen y colaboren en el proceso de educación de las adolescentes, promoviendo el acceso a información sobre métodos anticonceptivos, conocimiento sobre las ETS y pautas sobre las consecuencias de un embarazo precoz para su vida familiar, escolar o profesional, reflexionando sobre proyectos personales.

Palabras clave: Sexualidad; Adolescencia; Embarazo en la adolescencia; Enfermedades sexualmente transmisibles.

\section{Introdução}

De acordo com a Organização Mundial da Saúde (2012), a adolescência é definida por um período que compreende a faixa etária entre 10 e 19 anos de idade, e pode ser definida como sendo a etapa da vida entre a infância e a idade adulta. Esta fase é caracterizada pelo início da puberdade, momento em que as mudanças fisiológicas e morfológicas começam a impor-se fortemente, afetando, sem dúvida alguma, o desenvolvimento da personalidade. É na fase da adolescência que o indivíduo procura a sua maturidade conquistando a sua própria e total autonomia (Freitas, 2003; Lira, Dimenstein, 2004).

Uma definição mais abrangente sobre a adolescência, segundo Sawyer et al. (2018), é essencial para o desenvolvimento adequado de leis, políticas sociais e sistemas de saúde a este público. É permeada por um complexo processo de crescimento e desenvolvimento no qual se atinge a maturidade física e a capacidade reprodutiva, sem, contudo, atingir a maturidade psicológica e social (Caminha et al., 2012).

Segundo estudos, a idade considerada média na qual os adolescentes estão entrando no processo de puberdade tem diminuído consideravelmente, atingindo seu limite inferior. Quanto mais cedo, o adolescente entrar na puberdade, mais cedo, acontecerá seu amadurecimento biológico que não necessariamente coincide com o amadurecimento cognitivo e emocional, o que se constitui, portanto, fator de risco para uma iniciação sexual prematura e suas negativas conseqüências (Boruchovich, 1992).

A questão referente à queda da idade média da menarca e do início da atividade sexual aparece entrelaçada à gravidez na adolescência, assim como a ausência de informação e a dificuldade de acesso aos métodos contraceptivos. Igualmente corrente é a assertiva de que a gravidez em mulheres menores de 20 anos tem incidência maior nas classes economicamente desfavorecidas (Gomes et al., 2002).

Os adolescentes possuem peculiaridades próprias de seu desenvolvimento psicoemocional, como a onipotência e desejo de experimentar situações novas e, por isso, tendem a adotar comportamentos de risco, tornando-se mais vulneráveis (Ferreira et al., 2017).

O Ministério da Saúde em seu Manual de Gestação de Alto Risco (2012), afirma que a adolescência, em si, não é fator de risco para a gestação. Há, todavia, possibilidade de risco psicossocial, associado à aceitação ou não da gravidez com reflexos sobre a vida da gestante adolescente que podem se traduzir na adesão (ou não) ao preconizado durante o acompanhamento pré-natal.

São altos os índices de gravidez precoce no Brasil, uma vez que 28 a 30\% dos recém-nascidos são filhos de mães com idade inferior a 19 anos. Segundo a pesquisa divulgada pelo Instituto Brasileiro de Geografia e 
Estatística (IBGE), o número de adolescentes com idade entre 10 a 14 anos que esperavam um filho ou estavam no pós-parto quase dobrou entre 2000 e 2002 (Santos, 2006).

O crescimento da concepção da liberação do comportamento social, em específico, o da sexualidade, contribui significativamente para o elevado aumento da gravidez no período da adolescência, devido ao desconhecimento do próprio corpo enquanto função reprodutora, vinda da ausência de uma educação esclarecedora tanto no âmbito familiar como no escolar e social (Bueno, 2006).

Do ponto de vista social e cultural, esses fatores podem associar-se às transformações dos costumes sexuais e à maciça exposição a mensagens sexuais nos meios de comunicação. Porém, a liberação dos costumes nem sempre vem acompanhada com informação necessária sobre as conseqüências da atividade sexual iniciada precocemente e sem proteção (Santos, 2006).

A gravidez na adolescência é considerada um problema de saúde pública mundial. Para um melhor entendimento dos possíveis fatores associados às gestações nessa faixa etária é necessário perceber a complexidade e a multicasualidade desses fatores, que tornam os adolescentes especialmente vulneráveis a essa situação (Lira; Dimenstein, 2004).

Este tipo de gravidez frequentemente vem acompanhado de dificuldades, que podem ocasionar um desvio ou transtorno para a vida do adolescente. Destacam-se os "riscos biopsicossociais" tanto para a mãe quanto para seus filhos (Brasil, 2000).

A utilização de métodos contraceptivos entre adolescentes são mais baixas de um modo geral (Halbe, 2000). A compreensão dos fatores associados a não- utilização de anticoncepcionais na adolescência é essencial, dada a importância que o uso desses métodos tem para a prevenção dos sérios problemas que afetam a vida sexual do adolescente. Portanto, um desafio colocado refere-se à implantação de ações educativas e preventivas de caráter contínuo e sistemático.

Diante da contextualização acerca da gravidez na adolescência como um problema social e de saúde pública, considerando as repercussões no enfrentamento da gestação na adolescência, questiona - se: quais as repercussões do enfrentamento gestacional para a mãe adolescente? Desta forma, é necessário subsidiar o planejamento de ações preventivas para a redução do número de mães adolescentes, afim de evidenciar comportamentos de risco em decorrência da gravidez em faixa etária precoce.

Este estudo tem como objetivo compreender as contribuições dos profissionais de saúde frente a educação sexual e reprodutiva das adolescentes.

\section{Metodologia}

O estudo trata-se de uma revisão integrativa de literatura, com abordagem qualitativa. A revisão integrativa é um método que tem como finalidade oferecer suporte para a tomada de decisão e a melhoria da prática clínica (Benefield, 2003), possibilitando a síntese do estado do conhecimento de um determinado assunto, além de apontar lacunas do conhecimento que precisam ser preenchidas com a realização de novos estudos. Este método de pesquisa permite a síntese de múltiplos estudos publicados e possibilita conclusões gerais a respeito de uma particular área de estudo (Polit e Beck, 2006).

A revisão integrativa da literatura consiste na construção de uma análise ampla da literatura, contribuindo para discussões sobre métodos e resultados de pesquisas, assim como reflexões sobre a realização de futuros estudos. O propósito 
inicial deste método de pesquisa é obter um profundo entendimento de um determinado fenômeno baseando-se em estudos anteriores (Broome, 2000). É necessário seguir padrões de rigor metodológico, clareza na apresentação dos resultados, de forma que o leitor consiga identificar as características reais dos estudos incluídos na revisão (Beyea e Nicoll, 1998).

Segundo Denzin e Lincoln (2006), a pesquisa qualitativa envolve uma abordagem interpretativa do mundo, o que significa que seus pesquisadores estudam as coisas em seus cenários naturais, tentando entender os fenômenos em termos dos significados que as pessoas a eles conferem.

Seguindo essa linha de raciocínio, Vieira e Zouain (2005) afirmam que a pesquisa qualitativa atribui importância fundamental aos depoimentos dos atores sociais envolvidos, aos discursos e aos significados transmitidos por eles. Neste sentido, esses autores afirmam que, esse tipo de pesquisa preza pela descrição detalhada dos fenômenos e dos elementos que o envolvem.

A pesquisa qualitativa tem o ambiente natural como sua fonte direta de dados e o pesquisador como seu principal instrumento; os dados coletados são predominantemente descritivos; a preocupação com o processo é muito maior do que com o produto; o significado que as pessoas dão às coisas e à sua vida são focos de atenção especial pelo pesquisador; e a análise dos dados tende a seguir um processo indutivo (Ludke e André, 1986).

O levantamento de conteúdo foi realizado entre os meses de junho a dezembro de 2020, por meio da buscativa de artigos indexados na Biblioteca Virtual em Saúde (BVS), e Scientific Electronic Library Online (SciELO), com o auxílio das seguintes bases de dados: PubMed (National Center for Biotechnology Information), Lilacs (Literatura Latino-Americana e do Caribe em Ciências da Saúde), Base de Dados de Enfermagem (BDENF), PEPSIC (Periódicos Eletrônicos em Psicologia).

No Portal da Biblioteca Virtual em Saúde (http://decs.bvs.br), foram localizados os seguintes Descritores em Ciências da Saúde: Sexualidade; adolescência; gravidez na adolescência; doenças sexualmente transmissíveis; foi utilizado o operador booleano AND entre os descritores citados.

Os critérios de inclusão para a seleção dos conteúdos foram, artigos na linguagem portuguesa, inglesa e espanhola. Publicados na íntegra de acordo com a temática referente à revisão integrativa, documentos, regulamentações, normativas de entidades de saúde acerca do tema, artigos, teses, e dissertações publicados nos referidos bancos de dados compreendendo os anos de 2000 a 2020.

Os critérios de exclusão foram, artigos que não tivessem relevância com a temática, materiais duplicados, incompletos, resumos, resenhas, debates, relato de caso, relato de experiência, publicados em anais de eventos e materiais indisponíveis na íntegra.

\section{Resultados e Discussão}

A partir da revisão de literatura e análise dos estudos indexados nas bases de dados eletrônicas, acerca da temática proposta, foram encontrados 155 estudos científicos, sendo que, apenas 44 estudos foram selecionados, 22 atenderam aos critérios de inclusão previamente estabelecidos, destes, 08 foram excluídos com base nos critérios de exclusão, restando 14 artigos para composição e análise do estudo. O fluxograma com o detalhamento das etapas de pesquisa está apresentado a seguir na Figura 1. 
Figura 1. Fluxograma de identificação e seleção dos artigos (2020).

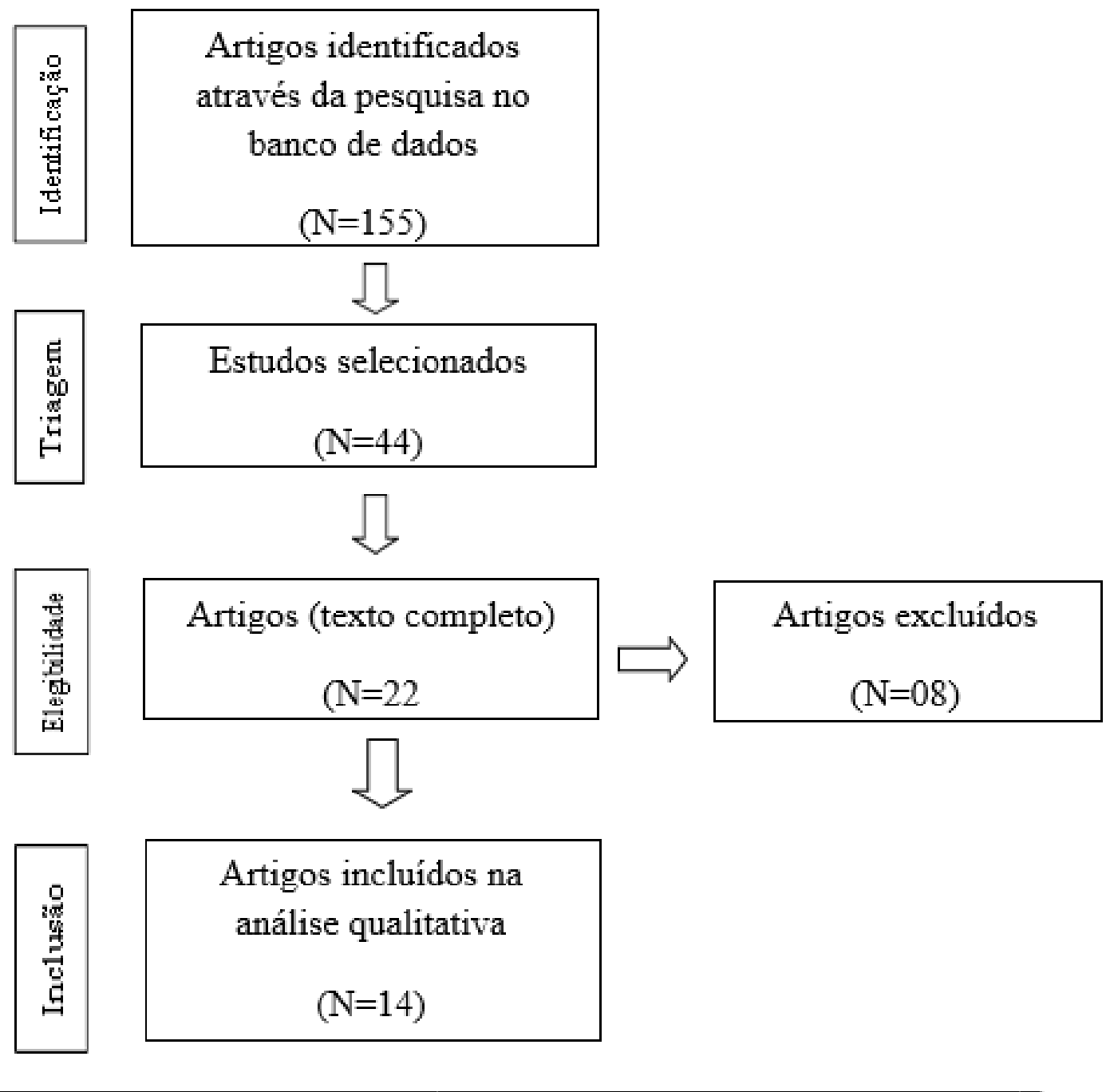

Fonte: Elaboração Própria (2020).

A fase da adolescência se constitui num período de transformações físicas e emocionais, sendo considerada desta forma, como um momento de inúmeros conflitos e de crises. Não se pode descrever a adolescência como uma simples adaptação às mudanças corporais, mas como uma importante fase no ciclo existencial da pessoa, uma tomada de posição social, familiar, sexual e entre o grupo (Bueno, 2006).

No início da adolescência, a ambivalência do jovem deriva em parte da ambigüidade ligada ao seu próprio corpo, como se ele não estivesse certo se deve agir como criança ou como adulto. Por outro lado, esta dificuldade é reforçada pelos próprios pais, que também se mostram inseguros com relação à posição do adolescente dentro do próprio desenvolvimento (Brasil, 2000).

O marco da adolescência se dá pelas lutas do indivíduo consigo mesmo e pelas contradições de atitudes, uma vez que o indivíduo busca uma definição da vida que tem pela frente; ou seja, o adolescente encontra-se numa procura a si mesmo (Freitas, 2003).

Segundo Santos (2006), a adolescência traz consigo o início da puberdade e o desenvolvimento das características sexuais, dos órgãos sexuais primários e secundários e um notável crescimento somático, os quais pressagiam o destino do 
corpo da criança em sua inevitável transformação. As transformações físicas acontecem devido ao elevado aumento da produção de hormônios neste período. Essas alterações hormonais e as eventuais incapacidades ou relutâncias em adaptar-se às alterações físicas contribuem também para alguns estados de depressão, característicos dos adolescentes. Alternadamente, se observam períodos de intensa energia física, entusiasmo e inquietação sem limites.

A inserção na puberdade precocemente, geralmente ocasiona um amadurecimento biológico que não necessariamente vai coincidir com o amadurecimento cognitivo e emocional, apresentando, portanto, como um fator de risco para um início da atividade sexual prematura e suas negativas conseqüências. As adolescentes do sexo feminino que entram na puberdade mais cedo do que a média, apresentam tendência maior a ter experiências sexuais precoces (Figueiredo, 2002).

A puberdade feminina apresenta seu início, geralmente, entre 11 e 14 anos, variando esse período de pessoa para pessoa. Em geral, a primeira menstruação (menarca) coincide com o surgimento de uma série de transformações do corpo que já se vinham manifestando na fase conhecida como pré-puberal. Consensual que a idade em que uma mulher atinge a puberdade é bastante variável e influenciada por fatores pessoais e ambientais. Não é incomum a puberdade começar um pouco mais cedo ou um pouco mais tarde que a média e entre os muitos fatores de influência, se inclui a hereditariedade, o grupo étnico, o tipo do corpo, nutrição, estilo de vida, toxinas ambientais, etc. (Bueno, 2006).

O surgimento da menarca marca o estágio do amadurecimento uterino e aponta que o corpo está pronto para a capacidade de reprodução, mas não significa que a adolescente tenha atingido o grau máximo de função reprodutiva (Freitas, 2003).

Desta forma, pode-se dizer que a puberdade é marcada por transformações significativas, tanto biológicas como psicológicas e sociais. A transformação biológica do ponto de vista cultural é a transformação do estado não reprodutivo ao reprodutivo (Bueno, 2006).

Na mulher, observa-se alargamento dos quadris e maior deposição de gordura, aparecimento de pelos pubianos e axilares, desenvolvimento mamário, menarca e início dos ciclos ovulatórios, com conseqüente capacidade reprodutiva. Mas a puberdade, não proporciona apenas mudanças físicas, mas, sobretudo, psicológicas. As alterações hormonais despertam a sensibilidade sexual e, conseqüentemente, é neste período que muitos adolescentes começam esporadicamente a ter relações sexuais (Bueno, 2006).

A vida sexual de todas as pessoas é formada por fases, marcadas por fatos que mostram o início e o término de mudanças significativas. Para a mulher, essas etapas são especialmente assinaladas por transformações orgânicas. A primeira menstruação, a primeira relação sexual, a primeira gravidez e a última menstruação evidenciam mudanças significativas que podem ser fontes de conflitos, ansiedades e inseguranças (Gomes, 2002).

De acordo com Saito (2003), a prática da sexualidade apresenta como base três condições que são mais relevantes, sendo elas: a histórica, a cultural e a de cunho social. Concordando com este autor, Bié, Diógenes e Moura (2006) consideram que, de acordo com o ponto de vista histórico, a visão da sexualidade no tempo ajuda no entendimento não como proposta individual, mas sim vinculada a relações de poder de ordem político-econômica, cultural, social, religiosa, moral, ética, subordinando o comportamento sexual do indivíduo a valores e instituições que envolvem de forma dinâmica cada época e que podem ser sob múltiplos aspectos transpostos para os dias de hoje.

A forma com a qual o adolescente vai lidar com comportamento sexual é resultado de fatores presentes na cultura e no ambiente que se vive, que cada vez mais erotiza o relacionamento social. Desta forma pode-se entender melhor o comportamento sexual do adolescente que muita vezes comporta-se por imitação e não pela modelação, o que resulta em consequiências reforçadoras como a gravidez na adolescência (Lira; Dimenstein, 2004).

A questão da influência da sociedade é determinante no comportamento sexual. É ela quem vai classificar este comportamento desde o "ficar" até o namorar. "Ficar" é um tipo de relacionamento íntimo sem compromisso de fidelidade 
entre os parceiros. Num ambiente social (festa, barzinho, boate) jovens sentem-se atraídos, dançam conversam e resolvem ficar juntos aquela noite. Nessa relação podem acontecer beijos, abraços, colar de corpos e até uma relação sexual completa, desde que ambos queiram (Bueno, 2006).

O impulso pelo adolescente, de efetuar a exibição de seus instintos, juntamente com a necessidade de provar a si mesmo sua virilidade e sua independente determinação em conquistar outra pessoa do sexo oposto, contraria com facilidade as normas tradicionais da sociedade e os aconselhamentos familiares e começa, avidamente, o exercício de sua sexualidade (Santos, 2006).

Mesmo com todo desenvolvimento social, cultural e tecnológico ocorrido no século XX, informações relacionadas aos aspectos de crescimento e desenvolvimento biopsicossocial e sexual, tão necessárias à construção da identidade psicossocial, não têm alcançado de forma ampla e adequada a maior parte dos adolescentes, ocasionando entre estes altos índices de desinformação sobre diferentes aspectos (Figueiredo, 2002).

As questões referentes à cultura, a vergonha e o preconceito dificultam o relacionamento entre pais e filhos quando o assunto é a sexualidade, e os pais, diante desses aspectos, condensam suas orientações em recados dados de maneira indireta, dificultando, assim, a compreensão destes pelos filhos (Sousa, 2005).

A gravidez na adolescência pode ser considerada uma consequiência da emissão de um comportamento de risco da adolescente, como manter relações sexuais sem medidas contraceptivas, utilizá-las inadequadamente ou iniciar precocemente a atividade sexual (Freitas, 2003).

O planejamento da gravidez na adolescência, na grande maioria, não é realizado, acontecendo em decorrência de uma atividade sexual não planejada e não protegida. A falta de conhecimento do funcionamento do próprio corpo, a falta de suporte afetivo dentro das famílias, a busca de reconhecimento e aprovação constantes por parte dos grupos de companheiros e a deficiência de programas adequados têm sido em grande parte os responsáveis pelas estatísticas alarmantes de gravidez na adolescência (Freitas, 2003).

Psicologicamente, as características gerais da adolescência (a curiosidade, o desejo de experimentação, o pensamento mágico, a tendência a transgredir as normas em busca de auto-afirmação, a grande intensidade emocional das paixões) contribuem por si mesmas, para tornar a adolescente mais vulnerável à gravidez não-planejada (Bueno, 2006).

Esses fatores gerais somam-se outros, de caráter circunstancial e pessoal. Muitas adolescentes engravidam para preencher vazios emocionais decorrentes da desestruturação familiar, da dificuldade de diálogo com os pais. Outras engravidam na tentativa de prender o parceiro ou de firmar a própria identidade. As adolescentes que iniciam a vida sexual precocemente ou engravidam nesse período, geralmente vêm de famílias cujas mães se assemelharam a essa biografia, ou seja, também iniciaram vida sexual precoce ou engravidaram durante a adolescência (Bueno, 2006).

Existem outras condições que também levam a este favorecimento, tais como: ausência de educação sexual nas escolas e de programas de planejamento familiar nos serviços públicos de saúde. As adolescentes grávidas estão inseridas num contexto de conflitos: criança ou mulher, filha ou mãe, não sabendo se comportar diante da gravidez e sem saber que atitude adotar diante da sociedade e consigo mesma (Brasil, 2000).

As consequências indesejáveis da gravidez no período da adolescência não são basicamente biológicas, mas, sobretudo as psicossociais, culturais e econômicas. Atualmente, a adolescência passou a ser considerada como período para a atividade escolar e para preparação profissional no contexto de dependência familiar, tanto econômica quanto emocional. Quando ocorre a gravidez a jovem tem de realizar, de uma só vez, tanto os ajustes exigidos por esse novo estado quanto os exigidos pela adolescência. E, naturalmente, quanto mais precoce a gravidez, maior a sobrecarga de conflitos a serem elaborados (Gomes, 2002). 
Quanto aos problemas biológicos podem ser apresentados pela adolescente grávida problemas de crescimento e desenvolvimento emocionais, além de complicações da gravidez e durante o parto. É por isso que alguns autores consideram a gravidez na adolescência como sendo uma das complicações da atividade sexual (Bueno, 2006).

O fato de se tornar mãe no começo da vida reprodutiva antecipa a maturidade biológica, e precipita momentos socialmente institucionalizados para a reprodução, com claras implicações para a constituição de família e a organização social dominante. As expectativas sociais diante da idade para o início da reprodução, no entanto, alteram-se cultural e historicamente, e a gravidez, no período modernamente chamado de adolescência, é abordada de modo diferente de décadas passadas (Brasil, 2000).

Para as mulheres, a gravidez representa uma intensa experiência orgânica e psicossocial, que determina transformações na imagem do corpo, no sistema hormonal, na identidade, no tipo de vínculo com o parceiro, no papel da sociedade e nas responsabilidades socioeconômicas. A gravidez pode levar a mulher a identificar-se com a criança e reviver o estado de dependência ou, ao contrário, levá- la a identificar-se com a figura materna (Gomes, 2002).

A gestação é acompanhada de riscos, sendo estes considerados uma grande preocupação brasileira. Um dos riscos que permeiam a gravidez é a gestação na adolescência, que coloca a adolescente vulnerável às patologias antes, durante e após o parto, incidindo não só na adolescente, como também no seu filho (Bueno, 2006).

O período da adolescência, por si só, é considerado uma fase de transformações e incertezas, pois a adolescente apresenta um corpo em mudanças, em desenvolvimento, com características sexuais adultas e ao mesmo tempo precisa adequar seu comportamento às novas exigências sociais e culturais que tal fase impõe. Assim, engravidar, nesta fase gera complicações, tanto no que diz respeito ao fator social como no fator biológico e delicado na vida de uma mulher: a adolescência e a gravidez (Lira; Dimenstein, 2004).

O alto crescimento por parte da sociedade, da liberação de certos comportamentos, em específico o sexual, leva ao favorecimento do aumento da gravidez no período da adolescência, em função do desconhecimento do próprio corpo enquanto função reprodutora (Bueno, 2006).

Nas últimas décadas, muitos países presenciaram uma mudança nas atitudes com relação à sexualidade na adolescência. A atividade sexual iniciada em idades mais jovens mostrou que a comunicação efetiva entre pais e filhos sobre questões sexuais pode deter a gravidez na adolescência (Brasil, 2000).

De acordo com Bueno (2006), as adolescentes cujas mães conversam sobre sexo possuem menor probabilidade de iniciar a atividade sexual precoce e engravidar.

O relacionamento entre mãe e filho, em especial na forma de se comunicarem pode ser considerado uma questão que irá contribuir para que a primeira relação sexual aconteça mais tarde. Outras investigações ainda indicam que pais que têm um maior conhecimento sobre questões sexuais tendem a discutir mais esses assuntos com seus filhos quando comparados a pais que não possuem esse conhecimento (Boruchovitch, 1992).

Em geral, o adolescente não adquire do meio familiar, as informações que englobam a saúde e, quando têm acesso, essas informaç̃oes são muitas vezes limitadas e inadequadas, provenientes de amigos, de pessoas pouco preparadas para essa função. A maior parte das informações disseminadas diz respeito ao uso de preservativos para prevenção de DSTs/AIDS; entretanto, o mecanismo de funcionamento do corpo relacionado à puberdade, maturação sexual, vivências e conflitos decorrentes do crescimento e da sexualidade, com efeito, poucos são abordados (Gomes, 2002).

Antes de se pensar em tratamentos, vacinas e antibióticos, é preciso um trabalho de educação bem estruturado, com base não somente na AIDS, mas para todas as DSTs. Principalmente aquelas com potencial para induzir a malignidade, como é o caso do papilomavírus humano (HPV), Hepatite B e Hepatite C e aquelas com complicações importantes como as infecções 
pela Neisseria gonorrhoeae e Chlamydia trachomatis que podem levar a infertilidade o que trás sérios problemas para o emocional feminino (Febrasgo, 2000).

Neste contexto Mühlbauer e Fujui (2008) enfatizam a necessidade e a importância de uma educação referente a prática sexual no período da adolescência, pois esta promove os valores e princípios que irão moldar as gerações futuras para uma maneira de 'estar na vida' mais confiante, positiva, segura, como também, mais ativa e participante. Enfocam a questão do planejamento familiar como uma importante atividade de saúde, cujo objetivo é proporcionar aos adolescentes informações e meios necessários para que possam decidir de forma livre e consciente o melhor método para prevenir a gravidez indesejada bem como as DST.

\section{Considerações Finais}

Este estudo confirmou a importância de compreende a fase entre a infância e a idade adulta, considerando a passagem por um processo com sucessivas modificações de crescimento e de amadurecimento biopsicossocial, em que o indivíduo se transforma física e emocionalmente, onde, muitas vezes, ocorre o início da vida sexual.

O processo de educação em saúde sexual, desta maneira, se mostra como componente de grande destaque para a prevenção das DST, pois divulga e estimula o sexo seguro e o sexo protegido.

Este estudo colocou em evidência a informação e o conhecimento inadequado dos adolescentes em relação às questões de sexualidade e prevenção da gravidez precoce. Observa-se a necessidade de uma educação sexual mais ampla com espaços de diálogos e discussões de dúvidas das adolescentes, tanto na escola, como no âmbito familiar.

Portanto, é de grande importância que ocorra a participação e cooperação dos profissionais de saúde no processo de educação das adolescentes, promovendo o acesso à informações referente à métodos contraceptivos, o não uso de drogas e bebidas alcoólicas quando estiverem em uso de anticontraceptivos, conhecimento sobre as DST e orientá-las que uma gravidez precoce tem consequências para a sua vida familiar e inserção na sociedade, seja na escola ou na vida profissional, repercutindo sobre os projetos pessoais.

\section{Referências}

Benefield, L. E. (2003). Implementing evidence-based practice in home care. Home Healthc Nurse. 21(12):804-811.

Beyea, S. C., \& Nicoll, L. H. (1998). Writing an integrative review. AORN J. Apr, 67(4):877-80.

Broome, M. E. (2000). Integrative literature reviews for the development of concepts. In: Rodgers BL, Knafl KA, editors. Concept development in nursing: foundations, techniques and applications. Philadelphia (USA): W.B Saunders Company. 231-50.

Ludke, M., \& André, M. E. D. A. (1986). Pesquisa em educação: abordagens qualitativas. Editora Pedagógica e Universitária.

Polit, D. F., \& Beck, C. T. (2006). Using research in evidence-based nursing practice. In: Polit, D. F. Beck, C. T. Editors. Essentials of nursing research. Methods, appraisal and utilization. Philadelphia (USA): Lippincott Williams \& Wilkins. p. 457-94.

Bié, A. P. A., Diógenes, M. A. R., \& Moura, E. R. F. (2006). Planejamento familiar: o que os adolescentes sabem? RBPS, 19 (3): $125-130$.

Boruchovitch, E. (1992). Fatores associados a não utilização de anticoncepcionais na adolescência. Revista Saúde Pública, 26(6).

Brasil. Ministério da Saúde. (2011). Capacitação: Educação e Promoção da Saúde no Contexto Escolar, 2007. Portal da Saúde - www.saude.gov.br.

Brasil. Ministério da Saúde. (2000). Gravidez na adolescência: desejo ou subversão. In: Ministério da Saúde. Prevenir é sempre melhor. Brasília: Ministério da saúde. 
Research, Society and Development, v. 10, n. 2, e20010211282, 2021

(CC BY 4.0) | ISSN 2525-3409 | DOI: http://dx.doi.org/10.33448/rsd-v10i2.11282

Bueno, G. M. (2006). Variáveis de risco para a gravidez na adolescência. <http://www.virtualpsy.org/infantil/gravidez.html>.

Febrasgo. (2000). Tratado de Ginecologia. Editores: Hildoberto Carneiro de Oliveira e Ivan Lemgruber. Revinter.

Figueiredo, A. C. (2002). Condições de vida e saúde reprodutiva de adolescentes na comunidade de Roda de fogo. Revista Brasileira Materno Infantil, Recife, 2(3), 291-302.

Freitas, F. et al. (2003). Rotinas de ginecologia. (4a ed.). Artmed.

Gomes, R. et al. (2002). A visão da pediatria acerca da gravidez. Revista Latino Americana de enfermagem. 10(3), 408 - 414.

Halbe, A. F. P. (2000). Contracepção e sexualidade da mulher. In: Halbe, H. W. Tratado de ginecologia. (3a ed.), Rocca

Lira, J. B., \& Dimenstein, M. (2004). Adolescentes avaliando um projeto social em uma unidade básica de saúde. Psicologia em Estudo, Maringá, 9(1), 37-45, jan/abr.

Mühlbauer, J. H., \& Fukui, A. M. (2011). O profissional de saúde e o planejamento familiar na adolescência. Uniandrade, 2008. <www.uniandrade. edu.br/links/menu3/.../revista.../artigo14.pdf>.

Passos, M. R. L. et al. (2011). Doenças Sexualmente Transmissíveis. Se educar, dá para evitar! Revinter.

PNDS. (2006). Pesquisa Nacional de Demografia e Saúde. <http:/bvsms.saude.gov.br/bvs/pnds/fecundidade.php>.

Saito, M. I. (2003). Sexualidade e educação sexual. In: MARCONDES, E. (Coord.). Pediatria básica. (9a ed.), SARVIER, 692-693.

Santos, M. M. J. F. (2006). Gravidez Precoce: matéria da capa. Estado de Minas, Belo Horizonte, p.4-5, 14 de maio.

Sousa, C. (2005). Gravidez na adolescência. In: Manual do Adolescente. <http://www.adolescente.psc.br/site/fiqueligado_int.php?i=49>.

OMS - Organização Mundial de Saúde (2009). Child and adolescent health and development. Genebra: OMS. http://www.who.int/child-adolescent-health/.

OMS - Organização Mundial de Saúde (2012). A gravidez na adolescência: Ficha informativa no. 364. http://www.who.int/mediacentre/factsheets /fs364/en /index.html. 